\title{
INVISIBILIDAD Y DESOBEDIENCIA CIVIL
}

\section{Germán Torres de Huertas}

Univ. Politécnica de Valencia. Máster Universitario en Artes Visuales y Multimedia (AVM)

\section{Resumen}

La instalación "Invisibilidad y Desobediencia Civil" investiga el concepto de invisibilidad, en relación con el pensamiento francés antiocularcentrista. Se trata de un sistema de lentes ópticas alienadas en paraxial, que practica la invisibilidad física sobre video proyecciones. El trabajo es una representación material, del efecto que la presencia o ausencia de la imagen puede ejercer en la construcción de nuestros imaginarios. Cuestión que ha ocupado la preocupación epistemológica sobre la supremacía de la visión en la tradición científica, y en los métodos históricamente usados para acceder al conocimiento experimental. La importancia que implica la invisibilización de determinadas imágenes, por el medio que sea, estriba en que define nuestras posiciones tanto en esa búsqueda del conocimiento, como en la configuración política de la sociedad, en el más amplio sentido.

\section{Palabras-clave: INVISIBILIDAD; ÓPTICA; PARAXIAL; ANTIOCULARCENTRISMO; PROYECCIÓN}

\section{INVISIBILITY AND CIVIL DISOBEDIENCE}

\section{Abstract}

The installation "Invisibility and Civil Disobedience" explores invisibility concept, in reference to the antiocularcentrism philosophical thought currents. over video proyection, using optical lenses aligned in paraxial. It's a system of optical lenses aligned in paraxial, which practices physical invisibility on video projections. The work is a symbolic materialization of the effect that can exert the image and its possible invisibility over the construction of our imaginaries and over the way of forming experiences and knowledge. A question that has occupied the epistemological concerns about the supremacy of vision on the scientific tradition and methods of access to experimental knowledge. The importance that imply to make invisibility some images, by whatever means, it's that defines our particular way in the pursuit of knowledge, as well as in the political configuration of the society, in the broadest sense of that word.

Keywords: INVISIBILITY; OPTIC; PARAXIAL; ANTIOCULARCENTRISMO; PROJECTION

\footnotetext{
Torres de Huertas, Germán. 2016. "Invisibilidad y desobediencia civil". AusArt 4 (1): pp-pp. 11-17 D0I: 10.1387/ausart.16672
}

\section{AUSART}




\section{INTRODUCCIÓN}

El interés del pensamiento antiocularcentrista por la eterna imposición de lo visible sobre lo oculto; de la presencia sobre la ausencia; de lo iluminado sobre lo que está en penumbra; de la plenitud sobre el vacío, radica en su preocupación por cómo esa preeminencia de la visión, ha condicionado el modo de construir el conocimiento y la experiencia en la tradición científica y filosófica occidentales, dejando fuera de la realidad todo lo que quedara "fuera del campo de la visión".

En la práctica, esas imposiciones del pensamiento y de la ciencia, influirían en el arte fomentando la hegemonía del espacio sobre el tiempo; del producto sobre el proceso; y de la forma sobre el sonido, entre otras consecuencias.

Decía Merleau-Ponty a propósito del modelo ocularcentrista impuesto: "El mundo percibido es el fundamento presupuesto siempre por toda racionalidad, por todo valor y por toda existencia" $(1964,186)$. Añorando una percepción de la realidad más amplia que la provista por el racionalismo y nuestros ojos.

La supuesta validez de la observación como evidencia científica sería también cuestionada, entre otros, por autores como Canguilhem, Bachelar o Foucault en distintos textos. Y posteriormente, Martin Jay escribiría: "Lo que vemos está mediado por la construcción cultural de nuestra percepción aparentemente natural" ([1993] 2007, 295). Sin duda Jay fue otra voz que vislumbró posteriores investigaciones neurológicas que acabarían por minar la confianza en lo percibido por nuestro sistema visual.

En función de esa tradicional concepción visual de la realidad, los ocularcentristas han justificado la inexistencia de lo no visto. Una inexistencia que la extensión de la tecnología ha hecho convivir con la sobreinformación, al sumergirnos en el flujo de transmisiones y comunicaciones que dicha tecnología amplifica. Donde todo aquello que no aparezca representado en algún medio, tampoco existe para los ojos de la mayoría. Y queda excluido de la construcción de nuestros idearios. Tal y como C. Gianetti (1996) advierte cuando habla del nuevo poder emergido desde la invisibilidad de lo virtual, que a la vez invisibiliza otras realidades.

Frente al ocularcentrismo, Martin Jay apuntala: "Siempre estamos en medio de un proceso formado por múltiples capas. [...] Lo visible y lo invisible son como un pliegue del Ser, un entrecruzamiento, un gozne" ([1993] 2007, 243).Pero 
aunque sea imposible negar hoy, la existencia de espectros de luz y sonido imperceptibles para nuestros sentidos, seguimos negando aquello que nuestros ojos no puedan consumir. $Y$ seguimos asintiendo en distinto grado a lo que por el ojo entra. Motivo suficiente para que el Poder se interese en administrar nuestra visualidad.

Si el cine para Barthes y Metz duplicaba la realidad en movimiento (Metz [1977] 1982, 14), el dispositivo de lentes en paraxial permite agujerear la proyección de esa duplicidad virtual de la realidad, para ver a través de ella otra realidad. Como si al someter la imagen proyectada al efecto de invisibilidad de las lentes, siguiéramos la intención de R. Barthes, de atender a la experiencia de la imagen eludiendo su posible "hipnosis", mirando por debajo de ella.

Siempre advertidos por Barthes de que: "La vista, al revelar un mundo de objetos opacos no puede conducir a la reflexión" ([1964] 1972, 92). Queda claro que el ojo no es prueba suficiente para el conocimiento de una realidad, más aun si consideramos las investigaciones de neurofisiólogos como Humberto Maturana, Rodolfo R. Llinás, Antonio Damasio o Von Helmholz, coincidentes todos en la permeabilidad de nuestra percepción visual a lo previamente conocido, y en el componente creativo del proceso visual en nuestro en nuestro cerebro.

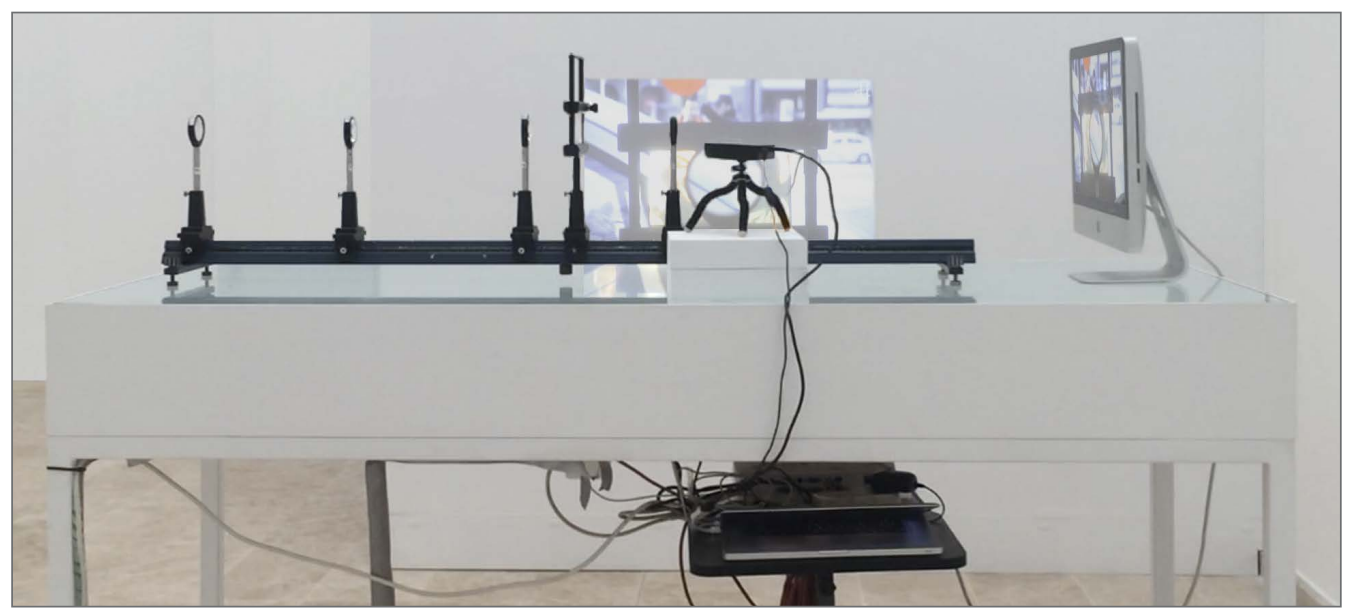

Fig. 1. Vista del Dispositivo de Invisibilidad. 


\section{INVISIBILIDAD Y DESOBEDIENCIA CIVIL}

La instalación propone invertir cierta invisibilidad impuesta por la nueva Ley Orgánica de Protección de la Seguridad Ciudadana de 2015. Dicha ley, limita el ejercicio de la libertad de prensa, penalizando la toma y publicación de imágenes no autorizadas de los cuerpos de seguridad del estado así como de autoridades, con multas de hasta 30.000 euros.

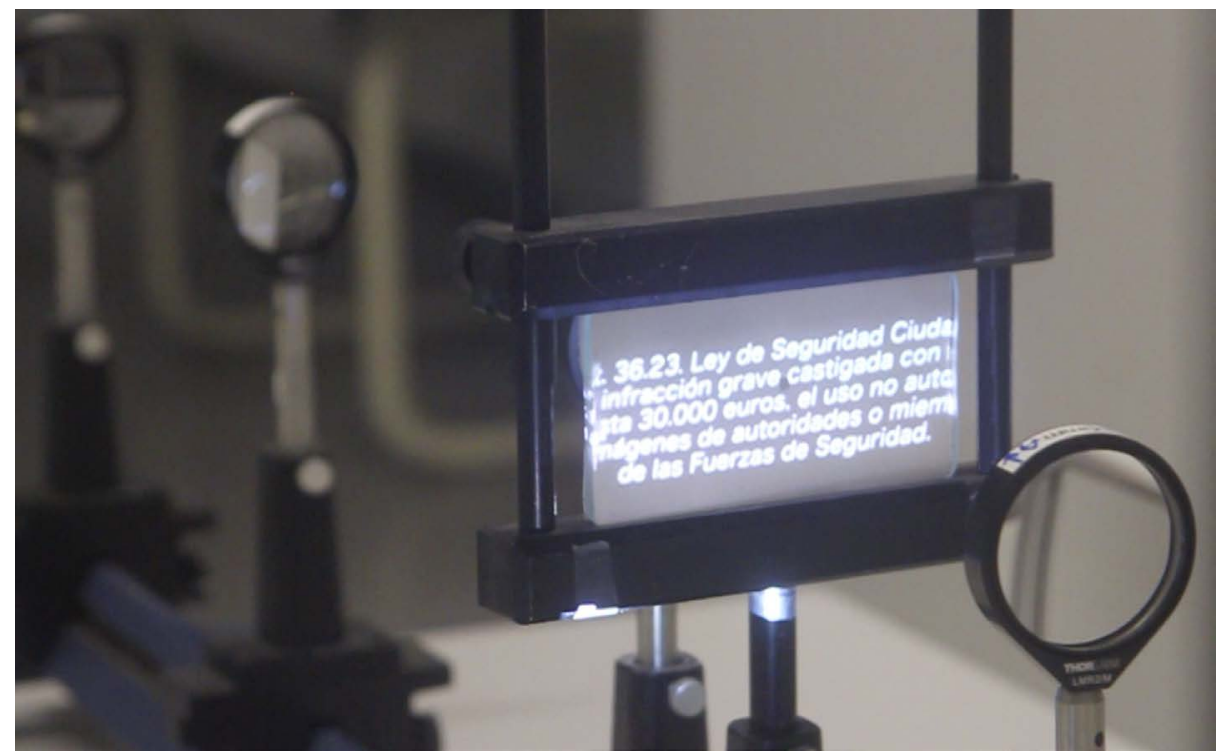

Fig. 2. Art. 36.23. Ley Organica de Protección de la Seguridad Ciudadana, proyectada sobre la ventana divisor de haces de luz.

El dispositivo, lo que invisibiliza son las imágenes proyectadas en una ventana divisor de haz de luz, referentes al texto de la ley que contiene la prohibición en su art. 36.23, así como imágenes de una realidad idealizada extraídas de la publicidad televisiva. De manera que resulte posible seguir viendo la realidad subyacente que tratan de solapar. Esa realidad está representada por un video de Amnistía Internacional (Robles González 2014), que recopila imágenes de agresiones policiales a periodistas en el ejercicio del derecho a la libre prensa. Y que el observador continúa viendo a través del efecto de invisibilización que el dispositivo ejerce sobre la imagen del texto de la ley. 


\section{DESCRIPCIÓN DEL DISPOSITIVO DE LENTES EN PARAXIAL}

El dispositivo, basado en el trabajo de J. S. Choi y J. C. Howell (2014) consiste en la alineación simétrica de cuatro lentes convergentes [3. B]. Se trata de lentes de $50,8 \mathrm{~mm}$. A diferencia del trabajo de Choi y Howel, el dispositivo combina dos lentes exteriores que son dobletes acromáticos de longitud focal f1 de $200 \mathrm{~mm}$. (a) y (b), con dos lentes centrales simples no acromáticas, de longitud focal f2 de $75 \mathrm{~mm}$. (c) y (d), montadas sobre un banco de ensayos ópticos.

El objetivo es hacer que la trayectoria de los haces de luz se curven al atravesar el sistema de lentes. Cuando la luz atraviesa la primera lente (a) sus rayos convergen antes de llegar a la segunda lente (c), que los colima hasta alcanzar la tercera óptica (d). Esta tercera lente converge de nuevo los haces de luz concentrándolos en un area de $0,4 \mathrm{~cm}$, de manera que el espacio alrededor resulta ignorado por la luz. Como un no lugar. $\mathrm{Al}$ ondular los rayos de luz le robamos la posibilidad de incidir sobre cualquier cosa que situemos alrededor del area de convergencia, no pudiendo refle-

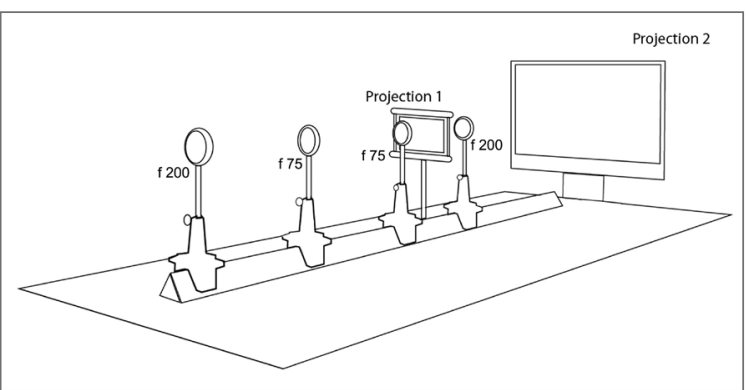

Fig. 3. Esquema del Dispositivo.

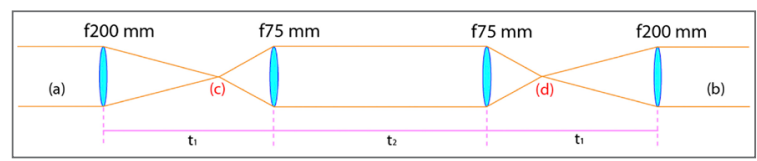

Fig. 4. Distancias Focales y Distancias Relativas de las lentes. jarse, que es lo que en la práctica inicia el proceso de nuestra visión.

La elección de lentes acromáticas atenúa las distorsiones de color. Y también incide en la corrección de las aberraciones esférica, coma, de astigmatismo, y en la deformación Petzval.

Para aumentar el diámetro visible de la última lente y evitar la percepción de su montura dentro del sistema, por parte del observador, es necesario alterar las distancias relativas de las lentes, desplazando las lentes exteriores hacia fuera. El efecto que se consigue de ese modo es prolongar la "pupila de salida", ocultando la visión las monturas de las lentes. Todo ello difiere de nuevo de los cálculos teóricos del trabajo de Choi y Howell. 
En la instalación, la imagen invisibilizada se presenta proyectada sobre una ventana divisor de haz de luz, que hace las veces de pantalla de proyección. La ventana refleja un $20 \%$ de la luz recibida y trasmite un $80 \%$. Está situada en el punto donde convergen la focalización de las lentes (d) y (b). Porque es donde se genera la pequeña área de invisibilidad de unos $4,5 \mathrm{~cm}$, dado el tamaño limitado de las lentes por su elevado coste.

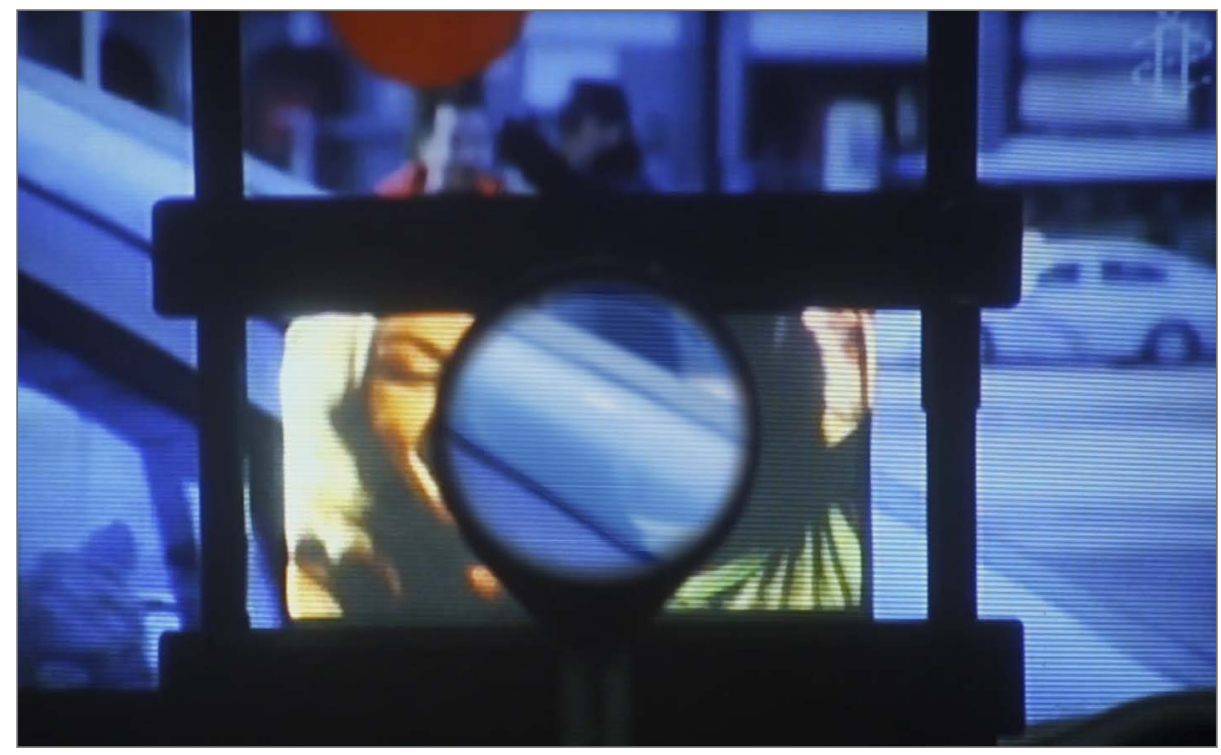

Fig. 5. Efecto final de invisibilidad sobre la proyección de video.

El dispositivo se completa con una video cámara, alineada con el eje óptico de las lentes y conectada en circuito cerrado a un proyector, para reproducir el efecto de invisibilidad ampliado en la pared contigua. La distancia de la cámara o punto de observación, hasta la primera lente, se calcula en relación al tamaño de las lentes, y su distancia focal y relativa.

El sistema óptico de lentes en paraxial, es multidireccional y permite despazar el ángulo de visión del observador en varios grados. A medida que el observador mueve la trayectoria de su vista fuera del eje óptico, la luz recibida pasa por un punto cercano, pero no exactamente por los puntos focales de las lentes. Por lo tanto, es importante no cegar la región trans-verso-real donde converge la luz, porque necesita pasar por algún lado o de lo contrario se reflejaría hasta el observador. 


\section{CONCLUSIONES}

Resulta tan posible ejercer la invisibilidad de una parte de la realidad, mediante un dispositivo simple de lentes ópticas, como a través de un dispositivo legal administrado por el Estado. Con diferentes consecuencias sobre nuestra percepción de esas realidades.

\section{Referencias}

Barthes, Roland. (1964) 1972. "There is no Robbe-Grillet School". En: Critical essays, translated from the French by Richard Howard. Evanston, IL: Northwestern University

Choi, Joseph S. \& John C. Howell. 2014 "Paraxial ray optics cloaking". Optics Express 22(24): 29465-78. http://arxiv.org/abs/1409.4705

Gianetti, Claudia. 1996. "Lo humano y lo invisible". Texto curatorial de la exposición internacional en Internet organizada por la Asociación de Cultura Contemporánea L'Angelot y Connect-Arte (Barcelona) http://www.artmetamedia.net/pdf/1Giannetti Humanolnvisible.pdf

Jay, Martin. (1993) 2007 Ojos abatidos : La denigración de la visión en el pensamiento francés del siglo XX. Traducción, Francisco López Martín. Madrid: Akal

Ley Orgánica 4/2015, de 30 de marzo, de Protección de la Seguridad Ciudadana (BOE núm. 77 , de 31 de marzo de 2015)

Merleau-Ponty, Maurice. 1964. The primacy of perception: And other essays on Phenomenological Psychology, the Philosophy of Art, History, and Politics. James M. Edie, ed. Evanston, IL: Northwestern University

Metz, Christian. (1977) 1982. The imaginary signifier: Psychoanalysis and the cinema. Translated by Celia Britton et al. Bloomington: Indiana University

Robles González, Juan Ramón. 2014. "\#derechoaprotestar - Grabar abusos policiales es arriesgado... ".Amnistía Internacional España. Video de Youtube, 2:51. Publicado el 4 nov. https://www.youtube.com/watch?v=OGbMtQjjEGY

\section{Notas}

Este proyecto ha sido posible gracias a la colaboración del Máster Universitario en Artes Visuales y Multimedia (AVM) de la UPV, los profesores Moisés Mañas y Salomé Cuesta; el Instituto de Ciencias de los Materiales de la UV, el profesor Fernando Sapiña-Navarro, Guillermo Muñoz Matutano, del GCOC-ITEAM de la UPV; Ios profesores Genaro Saavedra Tortosa y Fernando Silva Vázquez; Marcos López García y Rubén Martínez Lorente, del Departamento de Física Óptica de la UV por su ayuda en el montaje y ajuste del dispositivo. 